\title{
An Improvement to Mathon's Cyclotomic Ramsey Colorings
}

\author{
Xiaodong $\mathrm{Xu}^{*}$ \\ Guangxi Academy of Sciences \\ Nanning, 530003, China \\ xxdmaths@sina.com \\ Stanisław P. Radziszowski ${ }^{\dagger}$ \\ Department of Computer Science \\ Rochester Institute of Technology \\ Rochester, NY 14623, USA \\ spr@cs.rit.edu
}

Submitted: Oct 28, 2008; Accepted: Dec 9, 2008; Published: Jan 7, 2009

Mathematics Subject Classification: 05C55

\begin{abstract}
In this note we show how to extend Mathon's cyclotomic colorings of the edges of some complete graphs without increasing the maximum order of monochromatic complete subgraphs. This improves the well known lower bound construction for multicolor Ramsey numbers, in particular we obtain $R_{3}(7) \geq 3214$.
\end{abstract}

\section{Introduction and Notation}

A $\left(k_{1}, k_{2}, \ldots, k_{m}\right)$-coloring, for integers $m, k_{i} \geq 1$, is an assignment of one of $m$ colors to each edge in a complete graph, such that it does not contain any monochromatic complete subgraph $K_{k_{i}}$ in color $i$, for $1 \leq i \leq m$. Similarly, a $\left(k_{1}, k_{2}, \ldots, k_{m} ; n\right)$-coloring is a $\left(k_{1}, \ldots, k_{m}\right)$-coloring of the complete graph on $n$ vertices $K_{n}$. Let $\mathcal{R}\left(k_{1}, \ldots, k_{m}\right)$ and $\mathcal{R}\left(k_{1}, \ldots, k_{m} ; n\right)$ denote the set of all $\left(k_{1}, \ldots, k_{m}\right)$ - and $\left(k_{1}, \ldots, k_{m} ; n\right)$-colorings, respectively. The Ramsey number $R\left(k_{1}, \ldots, k_{m}\right)$ is defined to be the least $n>0$ such that

\footnotetext{
* partially supported by the National Science Fund of China (60563008) and the Basic Research Fund of Guangxi Academy of Sciences (080414)

${ }^{\dagger}$ corresponding author
} 
$\mathcal{R}\left(k_{1}, \ldots, k_{m} ; n\right)$ is empty. In the diagonal case, where $k_{1}=\ldots=k_{m}=k$, we will use simpler notation $\mathcal{R}_{m}(k)$ and $\mathcal{R}_{m}(k ; n)$ for sets of colorings, and $R_{m}(k)$ for the Ramsey numbers.

In the case of 2 colors $(m=2)$ we deal with classical graph Ramsey numbers, which have been studied extensively for 50 years. Much less has been done for multicolor numbers $(m \geq 3)$. A related area of interest has been the study of generalized Ramsey colorings, wherein the forbidden monochromatic subgraphs are not restricted to complete graphs. The second author maintains a regularly updated survey [2] of the most recent results on the best known bounds on various types of Ramsey numbers.

The next section shows how to improve on the well known construction by Mathon [1] for establishing lower bounds for $R_{m}(k)$.

\section{Extending Mathon's Construction}

In 1987, Mathon [1] gave a very elegant algebraical construction of certain $m$-class cyclotomic association schemes over finite field $\mathcal{F}_{p}$, which when interpreted as $m$-colorings of the edges of $K_{p}$ and $K_{m(p+1)}$ give constructive lower bounds for the corresponding classical diagonal Ramsey numbers. Specifically, for a prime power $p=m t+1$ with even $t$, one considers the basic $m$-th residue graph $H_{p}^{m}$ with vertices in $\mathcal{F}_{p}$ and $\{x, y\}$ an edge if for some $0 \neq z \in \mathcal{F}_{p}, x-y=z^{m}$, Then, if $\alpha$ is the order of the maximum clique in $H_{p}^{m}$, the construction gives $m$-colorings of the edges of $K_{p}$ and $K_{m(p+1)}$ with the orders of the maximum monochromatic cliques equal to $\alpha$ and $\alpha+1$, respectively.

In the case of quadratic $(m=2)$ cyclotomic relations Mathon's construction is equivalent to the "doubling" of Paley graph described independently by Shearer [3], which, directly and indirectly, led to several best known lower bounds for Ramsey numbers (cf. [4]).

Higher order $(m \geq 3)$ cyclotomic relations beyond the basic $H_{p}^{m}$ so far seem to be not much exploited in the context of Ramsey constructions. Here, our main interest is in the Mathon's cubic association scheme (also pointed to, but not analyzed, by Shearer [3]). We show how to improve on Mathon's scheme in the case of cubic residues in $\mathcal{Z}_{p}$ for $K_{3 p+3}$, though as remarked at the end, a similar improvement holds for all $\mathcal{F}_{p}$ and $m \geq 2$. In the following we show how to include three additional vertices and obtain a 3 -coloring of the edges of $K_{3 p+6}$ without increasing the order of monochromatic complete subgraphs.

We begin with a description of Mathon's construction instantiated for 3 colors over $\mathcal{Z}_{p}$. Let $p$ be a prime of the form $p=3 t+1$ with even $t$, and let $\beta$ be a primitive element (generator) of $\mathcal{Z}_{p}^{*}$. The condition $p \equiv 1 \quad(\bmod 6)$ implies that $-1 \equiv\left(\beta^{q / 2}\right)^{3} \quad(\bmod p)$ is a cubic residue, which is needed for the associated coloring to be well defined. Consider 3coloring $H_{p}^{3}$ with the vertex set $\mathcal{Z}_{p}$, where the edge $\{x, y\}$ has color of the cubic character of $x-y$ in $\mathcal{Z}_{p}^{*}$, i.e. $\{x, y\}$ has color $i \in\{0,1,2\}$ if and only if $x-y \equiv \beta^{3 s+i}(\bmod p)$, for some integer $s$. It is well known that the subgraphs induced in $H_{p}^{3}$ by the three colors 
are isomorphic to each other [1]. Let $\alpha_{p}$ denote the order of the largest monochromatic clique in $H_{p}^{3}$.

Next, we "triple" the coloring $H_{p}^{3}$ to the coloring $M_{p}$ on the vertex set $X=U \cup V$ of $3 p+3$ vertices, where $U, V \subset \mathcal{Z}_{p} \times \mathcal{Z}_{p},|U|=3,|V|=3 p$, and $U$ and $V$ are defined by

$$
\begin{aligned}
& U=\left\{u_{0}, u_{1}, u_{2}\right\}=\left\{(0,1),(0, \beta),\left(0, \beta^{2}\right)\right\}, \\
& V=V_{0} \cup V_{1} \cup V_{2}, \text { where } V_{i}=\left\{\left(\beta^{i}, a\right) \mid a \in \mathcal{Z}_{p}\right\}, \text { for } i \in\{0,1,2\} .
\end{aligned}
$$

Each edge $e=\{(x, y),(s, t)\}$ in $M_{p}$ is colored according to the cubic character of $x t-y s$ in $\mathcal{Z}_{p}$. If $x t-y s=0$ then $e$ has the special color $c \notin\{0,1,2\}$ (which later will be recolored), otherwise $e$ has color $i \in\{0,1,2\}$ if and only if $x t-y s \equiv \beta^{3 s+i}(\bmod p)$, for some integer $s$.

The main result related to this construction obtained by Mathon [1] is that the order of any monochromatic clique in $M_{p}$ is at most $\alpha_{p}+1$. In addition, the coloring $M_{p}$ satisfies the following properties:

A. Color $c$ induces $p+1$ vertex disjoint triangles, $U$ is one of them. For each $i, j \in\{0,1,2\}$, $u_{i}$ 's neighborhood in color $j, N_{j}\left(u_{i}\right)$, is $V_{j+1}(\bmod 3)$.

B. $M_{p}$ is vertex transitive, and colors $\{0,1,2\}$ induce isomorphic colorings. Thus, each vertex $x \in X$ has degree $p$ in each color $i \in\{0,1,2\}$, and color $i$ neighborhood of $x$, $N_{i}(x)$, induces a coloring isomorphic to $H_{p}^{3}$.

C. If the edge $\{x, y\}$ has the special color $c$, then $N_{i}(x) \cap N_{i}(y)=\emptyset$ for all $i$ in $\{0,1,2\}$. Consequently, after an arbitrary recoloring of the edges from color $c$ to colors $\{0,1,2\}$, any monochromatic clique in $M_{p}$ contains at most one vertex from $U$ if it contains any vertices not in $U$.

Theorem 1 For prime $p \equiv 1 \quad(\bmod 6)$, let $\alpha_{p}$ denote the order of the largest monochromatic clique in the cubic residues coloring $H_{p}^{3}$. If $k=\alpha_{p}+2 \geq 4$ then $R(k, k, k)>3(p+2)$.

Proof. We will extend the coloring $M_{p}$ described above (isomorphic to the construction by Mathon [1] for $m=3$ ) by three additional vertices to $M_{p}^{\prime}$, without incrementing the order of the largest monochromatic complete subgraph. We define 3-coloring $M_{p}^{\prime}$ of the edges of the complete graph on the vertex set $X \cup W=U \cup V \cup W$, where $W=\left\{w_{0}, w_{1}, w_{2}\right\}$, and $X, U, V$ are as before. Figure 1 gives the colors of the edges.

The middle $3 p \times 3 p$ section of the matrix with $\mathrm{x}$ 's is defined by the starting coloring $M_{p}$, while other entries in the rows/columns corresponding to $V_{i}$ mean that all the edges adjacent to this set have the same color as indicated in the matrix.

Let $S$ be the maximum order clique in $M_{p}^{\prime}$ in color $i$. If $S \cap W=\emptyset$ then $S$ is restricted to original $M_{p}$, so $|S| \leq \alpha_{p}+1$. One can easily check in Fig. 1 that every monochromatic triangle can have at most one vertex in the set $U \cup W$, so we can assume that $S \cap U=\emptyset$ 
but there exists $x \in S \cap W$. By properties $\mathbf{B}$ and $\mathbf{C}$ of $M_{p}$ we have $S \backslash\{x\} \subset V_{j}$, so again $|S| \leq \alpha_{p}+1 \cdot \diamond$

$$
\begin{array}{ccc}
\text { U } & \text { V } & \text { W } \\
012 & 012 & 012
\end{array}
$$

\begin{tabular}{|c|c|c|c|}
\hline $\mathrm{u} 0$ & 01 & 120 & \\
\hline $\mathrm{u} 1$ & 02 & 201 & \\
\hline $\mathrm{u} 2$ & 12 & 012 & \\
\hline V0 & 120 & $\mathrm{xxx}$ & \\
\hline V1 & 201 & $\mathrm{xxx}$ & \\
\hline V2 & 012 & $\mathrm{xxx}$ & \\
\hline w0 & 021 & 102 & \\
\hline w1 & 102 & 021 & \\
\hline רה & 210 & 210 & \\
\hline
\end{tabular}

Figure 1. Extending coloring $M_{p}$ by vertices $\left\{w_{0}, w_{1}, w_{2}\right\}$ to $M_{p}^{\prime}$.

The next corollary improves on the old bound 3211 by Mathon [1]. The new bound was not published, though it was already cited as an unpublished result in the survey [2].

Corollary $R(7,7,7) \geq 3214$.

Proof. For prime $p=1069$, it is known that $\alpha_{p}<6$ [1]. The bound 3214 follows from the Theorem. $\diamond$

One can similarly improve Shearer/Mathon's construction on $K_{m p+m}$ for other values of $m$, by producing $m$-colorings of $K_{m p+m}$. Note that for $m=2$ there is no improvement, and the case $m=3$ is that of the Theorem. For general $m$ with $\alpha_{p}+1 \geq m$, after chosing the set $U$ (now of $m$ vertices) we add a new set of vertices $W$ so that $|U \cup W|=m$ !. With each $x \in Y=U \cup W$ we associate a permutation $\left(i_{0}, \cdots, i_{m-1}\right)$ and color all the edges from $x$ to $V_{i_{j}}$ in color $j$. For $x, y \in Y$ the edge $\{x, y\}$ has color equal to the minimal index of the position at which the corresponding permutations are different. We omit the details since we don't know any specific parameters with $m>3$ for which this would improve on a best known published lower bound as in the Corollary. 


\section{References}

[1] R. Mathon, Lower Bounds for Ramsey Numbers and Association Schemes, Journal of Combinatorial Theory, Series B, 42 (1987) 122-127.

[2] S. P. Radziszowski, Small Ramsey Numbers, Electronic Journal of Combinatorics, Dynamic Survey 1, revision \#11, August 2006, 60 pages, http://www . combinatorics.org.

[3] J. B. Shearer, Lower Bounds for Small Diagonal Ramsey Numbers, Journal of Combinatorial Theory, Series A, 42 (1986) 302-304.

[4] Xu Xiaodong, Xie Zheng, G. Exoo and S. P. Radziszowski, Constructive Lower Bounds on Classical Multicolor Ramsey Numbers, Electronic Journal of Combinatorics, \#R35, 11(1) (2004), 24 pages, http://www.combinatorics.org. 